\title{
Correlation properties of spontaneous motor activity in healthy infants: a new computer-assisted method to evaluate neurological maturation
}

\author{
Sandra Waldmeier $\cdot$ Sebastian Grunt $\cdot$ \\ Edgar Delgado-Eckert • Philipp Latzin • \\ Maja Steinlin · Katharina Fuhrer · Urs Frey
}

Received: 18 July 2012 / Accepted: 26 March 2013 / Published online: 28 May 2013

(C) Springer-Verlag Berlin Heidelberg 2013

\begin{abstract}
Qualitative assessment of spontaneous motor activity in early infancy is widely used in clinical practice. It enables the description of maturational changes of motor behavior in both healthy infants and infants who are at risk for later neurological impairment. These assessments are, however, time-consuming and are dependent upon professional experience. Therefore, a simple physiological method that describes the complex behavior of spontaneous movements (SMs) in infants would be helpful. In this methodological study, we aimed to determine whether time series of motor acceleration measurements at 40-44 weeks and 50-55 weeks gestational age in healthy infants exhibit fractal-like properties and if this self-affinity of the acceleration signal is sensitive to maturation. Healthy motor state was ensured by General Movement assessment. We assessed statistical persistence in the acceleration time series by calculating
\end{abstract}

Sandra Waldmeier, Sebastian Grunt has been contributed equally.

S. Waldmeier $(\varangle) \cdot$ S. Grunt $\cdot$ M. Steinlin

Department of Pediatric Neurology, University Children's

Hospital Bern, PO-Box CH-3010, Bern, Switzerland

e-mail: sandrawaldmeier@hotmail.com

S. Grunt

e-mail: sebastian.grunt@insel.ch

E. Delgado-Eckert · U. Frey

University Children's Hospital, Basel, Switzerland

P. Latzin

Division of Respiratory Medicine, Department of Pediatrics,

University Hospital of Bern, Bern, Switzerland

K. Fuhrer

Department of Pediatrics, University Hospital, Fribourg,

Switzerland the scaling exponent $\alpha$ via detrended fluctuation analysis of the time series. In hand trajectories of SMs in infants we found a mean $\alpha$ value of 1.198 (95\% CI 1.167-1.230) at $40-44$ weeks. Alpha changed significantly $(p=0.001)$ at 50-55 weeks to a mean of 1.102 (1.055-1.149). Complementary multilevel regression analysis confirmed a decreasing trend of $\alpha$ with increasing age. Statistical persistence of fluctuation in hand trajectories of SMs is sensitive to neurological maturation and can be characterized by a simple parameter $\alpha$ in an automated and observerindependent fashion. Future studies including children at risk for neurological impairment should evaluate whether this method could be used as an early clinical screening tool for later neurological compromise.

Keywords Motor development in infants · Spontaneous movements · Detrended fluctuation analysis · Fractals correlation properties

\section{Introduction}

The quality of spontaneous motor activity in infants has been shown to predict later neurological outcomes (Burger and Louw 2009; Cioni et al. 1997; Einspieler et al. 2004; Einspieler and Prechtl 2005; Ferrari et al. 1990; Spittle et al. 2008). Spontaneous motor activity in infants has been studied with qualitative and quantitative methods. Prechtl and coworkers developed a qualitative approach to evaluate the motor integrity of infants based on the observation of spontaneous motor activity (Einspieler et al. 2004; Einspieler and Prechtl 2005). They described a method to assess the quality of movement patterns in infants and introduced the term "General Movements" (GMs), which describes whole-body movements characterized by changing speed, 
amplitude, and sequence (Cioni et al. 1997; Einspieler et al. 2004; Einspieler and Prechtl 2005; Ferrari et al. 1990). GMs are observed in newborns and young infants up to an age of 20 weeks post term. In premature infants and neonates (up to about 4 weeks post term), GMs appear more proximal and are characterized by small to moderate amplitude and slow to moderate speed (Writhing Movements $=\mathrm{WMs}$ ). At an age of 6-8 weeks post term, the movements become more distal, circular, have a smaller amplitude, show variable acceleration of the neck, trunk, and extremities in all directions and are characterized by a constant fluency (Fidgety Movements = FMs). The transition from WMs to FMs indicates a changing maturational stage.

Although this quality assessment allows the prediction of later neurological outcome, the method is time-consuming, depends largely on professional experience and shows a considerable intra- and inter-observer variability (Bernhardt et al. 2011). As such, there is a need for simple, observerindependent, physiological methods that can describe the complex behavior of SMs in infants during development. Various studies have investigated quantitative approaches to describe the trajectories of SMs in infants (Disselhorst-Klug et al. 2012; Karch et al. 2008, 2010; Meinecke et al. 2006; Piek 2001; van der Heide et al. 1999; Vaal et al. 2000). With the aid of three-dimensional movement analysis, the trajectories of SMs in infants were described, and it was possible to provide an objective description of age-dependent developmental steps in spontaneous motor behavior (Disselhorst-Klug et al. 2012). However most of these studies used complex equipment, and therefore the methods used are not necessarily suitable for a clinical setting.

Recent work used newer mathematical methods derived from nonlinear physics to quantify complex biological regulatory systems. In many complex systems in nature, longrange correlation of fluctuating signals has been described by detrended fluctuation analysis (DFA), a method to identify fractal-like properties of nonstationary time series that arise from stochastic processes. Signals in nature are influenced by external inputs which cause a certain trend with systematic deviation of mean value. DFA provides an observer-independent scaling exponent that describes the correlation properties of the regulation of physiological systems (Peng et al. 1993). Different studies have used DFA to describe correlation properties of physiological time series (Thamrin et al. 2010), including heartbeat (Goldberger et al. 2002; Peng et al. 1993, 1995), breathing (Baldwin et al. 2004; Cernelc et al. 2002; Thamrin and Frey 2009), temperature regulation (Stern et al. 2009) and gait control (Hausdorff et al. 1995, 1996; Dingwell and Cusumano 2010). Many regulatory systems have also been examined in infants (Baldwin et al. 2004; Cernelc et al. 2002; Stern et al. 2009). Based on DFA, these regulatory systems were thought to exhibit fractal-type long-range correlations
(Thamrin and Frey 2009; Thamrin et al. 2010), which are related to the characteristics and the determinism of the control mechanism and are also responsive to maturation.

According to Maraun et al. (2004), DFA is sensitive but not specific concerning long memory. Short-range correlated processes may yield equivalent results as longrange correlated processes, and therefore, results of DFA may be falsely positive (Maraun et al. 2004; Dingwell and Cusumano 2010). Instead of interpreting the results of DFA as long-range correlations (Peng et al. 1993), a different interpretation has been proposed, namely, as statistical "persistence" or "anti-persistence" in time series (Dingwell and Cusumano 2010; Maraun et al. 2004). "Persistence" means that deviations in time series are statistically more likely to be followed by subsequent deviations in the same direction. "Anti-persistence" means that deviations in one direction are statistically more likely to be followed by subsequent deviations in the opposite direction. For example, in gait control, statistically persistent fluctuation has been described as a critical marker of "healthy" physiological function (Dingwell and Cusumano 2010).

Nonlinear mathematical models have also been used to describe arm and leg movements in preterm infants (Ohgi et al. 2007, 2008; Gima et al. 2011). Ohgi et al. (2008) identified evidence of nonlinearity in spontaneous movements of premature infants by using three-dimensional accelerometer measurements of the infant's right hand. This study found that movements of infants with brain injuries were more unstable and less predictable than movements of low-risk children. In another study based on accelerometer measurements of leg movements, Gima et al. (2011) described the development of nonlinear characteristics in the spontaneous motor activity of healthy infants.

In our methodological study, we aimed to determine whether statistical persistence properties described by DFA exist in time series of accelerometer measurements in upper limb movements of healthy infants and whether these significantly change at two distinct maturational stages (MSs).

\section{Methods}

\section{Study design}

We quantified the variability and spontaneous movement ordering in one-dimensional acceleration (acc) of an infant's hand by measuring time series and determining the parameter $\alpha$, which characterizes statistical persistence properties in upper limb movements. Through a cross-sectional design, one-dimensional hand acceleration in infants at 40-44 gestational weeks (MS I, displaying WMs according to Prechtl) and in infants at 50-55 gestational weeks (MS II, displaying FMs according to Prechtl) was measured. 
Subjects

Twenty-two healthy neonates [11 boys and 11 girls; mean gestational age at birth 39.7 weeks (37.9-41.6 weeks, SD 1.1 weeks), mean birth weight $3,360 \mathrm{~g}(2,655-4,190 \mathrm{~g}$, SD $381 \mathrm{~g})$ ] were recruited between October 2002 and February 2005 from the maternity ward of the Department of Obstetrics and Gynecology, University Hospital, Bern.

We performed 25 hand acceleration measurements in MS I [mean age at measurement 41.8 weeks (39.943.4 weeks, SD 1.2 weeks)] and 19 measurements in MS II [mean age 52.7 weeks (50.0-55.5 weeks, SD 2.1 weeks)]. To establish the repeatability of the method, we achieved double measurements within 14 days and the same maturational stage in 12 infants. Five infants were longitudinally measured during both maturational stages. Detailed information regarding all measurements is provided in Table 2.

Exclusion criteria for the study were as follows: (1) complications during pregnancy (such as eclampsia, HELLP syndrome, premature contractions, vaginal bleeding, TORCH infections, maternal drug abuse), (2) neonatal conditions that could have a negative influence on the developmental course of the child (such as perinatal infections, umbilical cord $\mathrm{pH}<7.15$, APGAR Score $<5$ at $5 \mathrm{~min}$, or birth deformities), (3) abnormal neurological examination and/or abnormal spontaneous motor activity according to the GM assessment defined by Einspieler and Prechtl (2005). None of the children received any medication. The parents of all children gave oral and written consent for their participation in the study. The study was approved by the ethics committee of the University Children's Hospital of Bern and the ethics committee of the State of Bern.

\section{Clinical assessment of spontaneous movements}

Spontaneous motor activity of the infants was videotaped in supine position according to the recommendations of Einspieler et al. (2004). Video recordings were performed when the children were in Behavioral State IV, as defined by Prechtl (1974) (open eyes, gross body movements, no crying or suckling movements, no hiccups, and no distraction). Interaction between child and environment was minimized. Mothers were told not to touch their child and to avoid face-to-face interaction. However, mothers were allowed to calm infants during longer episodes of crying. If necessary infants could also be taken in their mother's arm or being breastfed for short periods. If the child was turning itself to one side, it was turned back in supine position. Parents and an observer who acted as a recording study nurse were present all the time of the measurement. Acceleration signals were recorded for a total duration of at least $1 \mathrm{~h}$, and a simultaneous video recording was realized during the whole measurement period. Within the video recording, an optimal sequence of 3-10 min in behavioral state IV (Prechtl 1974) was selected. A pediatric neurologist who was trained in the interpretation of spontaneous motor activity in infants classified GMs into different types of movement patterns according to Prechtl's definitions (Einspieler et al. 2004). Children showing pathological GMs at any maturational stage were excluded from data analysis.

Accelerometer measurement of hand movements

A prototype accelerometer (Valuline ${ }^{\mathrm{TM}}$ Accelerometer 7596-2, Pat. Nos. 457 4327, ENDEVCO, California, USA) with a weight of 10 grams was fixed to the infant's hand with a tape. The sensor was chosen because of its low weight. We sampled at $32 \mathrm{~Hz}$ and low-pass filtered at $10 \mathrm{~Hz}$, with an accuracy of 16 bits. According to the recommendations of Einspieler et al. (2004), GMs need to be observed over a time period of more than 3-5 min at least, in order to have a reproducible estimate of motor activity. As feeding times and sleep periods in the first months of life are difficult to anticipate, we measured a time period of at least $1 \mathrm{~h}$ to ensure sufficient movement activity in behavioral state IV (Prechtl 1974). According to Holger Kantz (personal communication 2012), 100,000 data points are the minimal allowable dataset for the estimation of correlation characteristics with DFA in a dataset of this type due to the relatively low measurement sampling frequency. Thus, we chose a measurement period of $1 \mathrm{~h}$ to deliver enough data points $(115,200)$. According to Ottaviano et al., only target-directed movements show right-hand dominance. In our study, target offering to the infants was minimized, and we did not insist fixing the accelerometer always to the same hand side (see Table 1). The accelerometer contained a mass body, which moved by accelerated hand movements and triggered a one-dimensional signal from the integrated sensor. The signal was transmitted through a thin, lowweight cable from the accelerometer to a personal computer. A representative example of the acceleration signal is illustrated in Fig. 1.

Data analysis: detrended fluctuation analysis

Typically in fractal-type time series, statistical properties of variations observed in a larger time window are similar to statistical properties of variations in a shorter time window (Fig. 1). This self-similarity can be quantified using DFA as described by Peng et al. (1993, 1995). Fluctuation analysis of accelerometer time series of each measurement was conducted using a custom-written software program implemented in the $\mathrm{R}$ environment [R Core Team (2012), http://www.R-project.org]. DFA yielded the presence of scaling, which was characterized by the slope alpha of a regression line fit. A detailed description 
Table 1 Patient characteristics

\begin{tabular}{lll}
\hline & Maturational stage I & Maturational stage II \\
\hline $\begin{array}{l}\text { Number of subjects } \\
\text { Gestational age at birth in }\end{array} \quad 16$ & 11 \\
$\quad$ weeks (SD, range) & $39.7(1.1 ; 37.9-41.6)$ & $39.8(1.2 ; 37.7-41.6)$ \\
Birth weight (g) (SD, range) & & \\
Birth length (cm) (SD, range) & $3360(381 ; 2655-4190)$ & $3362(256 ; 2905-3770)$ \\
Gender & $49.3(1.9 ; 46-53)$ & $49.4(1.7 ; 46-52)$ \\
& Male: $8(50 \%)$ & Male: $5(45 \%)$ \\
Age at measurement in weeks (SD, range) & Female: $8(50 \%)$ & Female: $6(55 \%)$ \\
Hand side of sensor application (number) & $41.8(1.2 ; 39.9-43.4)$ & $52.7(2.1 ; 50.0-55.5)$ \\
& Left: $17(68 \%)$ & Left: $14(74 \%)$ \\
& Right: $8(32 \%)$ & Right: $5(26 \%)$ \\
\hline
\end{tabular}

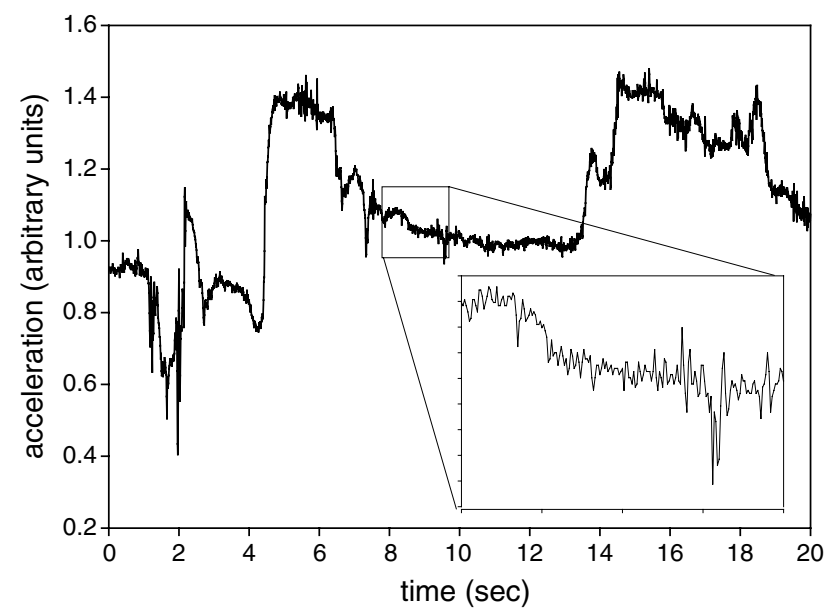

Fig. 1 Example of an acceleration signal. Acceleration signal sampled at a rate of $32 \mathrm{~Hz}$. Signal variations in a larger time window were statistically similar to signal variations in a shorter time window (fractal-type time series)

of DFA and the calculation of alpha can be found in the "Appendix 1".

Statistical tests using surrogate time series

To test the hypothesis that the participants controlled their movements by sending signals to their muscles that are temporally independent from previous signals, surrogates obtained via random shuffling of the original time series were used according to Dingwell and Cusumano (2010). The distribution of alpha values in these surrogate time series allowed for the rejection of this hypothesis for each participant (see "Appendix 2" for more details).

Dingwell and Cusumano (2010) also evaluated phaserandomized surrogates preserving the power spectra and auto-correlation properties of original time series in gait analysis (walking on a treadmill). According to their results, the hypothesis cannot be rejected that subjects independently choose their stride-time and stride-length as temporally correlated auto-regressive (AR) process or more generally as nearly equivalent auto-regressive moving-average (ARMA) process.

In the present study, we investigated to what extent this result applies to time series of motor acceleration measurements in healthy infants (at 40-44 weeks and 50-55 weeks gestational age). We found analogous properties of the motor acceleration time series.

More details on the generation of surrogate time series and the statistical analysis involving those can be found in "Appendix 2".

All calculations were conducted in $\mathrm{R}$ [R Core Team (2012)] using a custom-written software program implemented in the $\mathrm{R}$ environment, and the packages "fractal" [Constantine and Percival (2012)] and "tuneR" [Ligges (2011)].

Sampling frequency issues during measurements

In order to study potential issues with the method of DFA that could arise from a low-sampling frequency during the measurement, we conducted a simulation study. We investigated the effect of applying DFA to a time series obtained from an "original" time series by sampling the time series with a lower sampling rate. This sampling procedure boils down to leaving out one or more consecutive values from the original time series in a periodic manner. Fortunately, the effect is consistent with direction, suggesting that the errors in the alpha values caused by a low-sampling rate during measurements should not jeopardize the validity of our main results. Further details concerning these sampling frequency issues are specified in "Appendix 3".

Statistical analysis

The data were tested for normality. The combined crosssectional and longitudinal dataset was analyzed using various techniques to ensure the robustness of the findings. First, simple cross-sectional group comparison at the 
Table 2 Measurements of alpha

\begin{tabular}{|c|c|c|c|c|c|c|c|c|c|}
\hline \multirow[t]{2}{*}{ Subject } & \multicolumn{2}{|l|}{ MS I } & \multicolumn{2}{|l|}{ MS II } & \multirow[t]{2}{*}{ Subject } & \multicolumn{2}{|l|}{ MS I } & \multicolumn{2}{|l|}{ MS II } \\
\hline & Alpha 1 & Alpha 2 & Alpha 3 & Alpha 4 & & Alpha 1 & Alpha 2 & Alpha 3 & Alpha 4 \\
\hline 1 & - & - & 1.120 & 1.087 & 12 & 1.158 & - & - & - \\
\hline 2 & 1.245 & 1.245 & - & - & 13 & 1.162 & 1.178 & 1.175 & 1.100 \\
\hline 3 & 1.156 & 1.192 & - & - & 14 & - & - & 1.204 & 1.150 \\
\hline 4 & 1.224 & 1.155 & 1.021 & - & 15 & 1.260 & - & & - \\
\hline 5 & 1.091 & 1.170 & - & - & 16 & - & - & 1.141 & 1.222 \\
\hline 6 & - & - & 0.859 & 0.959 & 17 & 1.2925 & & 1.130 & - \\
\hline 7 & 1.097 & - & - & - & 18 & - & - & 0.957 & 1.020 \\
\hline 8 & 1.228 & - & - & - & 19 & 1.211 & 1.1729 & - & - \\
\hline 9 & 1.361 & 1.116 & - & - & 20 & 1.3722 & - & 1.214 & - \\
\hline 10 & 1.140 & 1.090 & - & - & 21 & 1.173 & - & 1.145 & 1.145 \\
\hline \multirow[t]{2}{*}{11} & - & - & 1.179 & 1.106 & 22 & 1.253 & 1.281 & - & - \\
\hline & \multicolumn{7}{|c|}{ MS I } & \multicolumn{2}{|c|}{ MS II } \\
\hline \multicolumn{3}{|l|}{ Mean alpha } & \multicolumn{3}{|c|}{1.198} & & & \multicolumn{2}{|c|}{1.102} \\
\hline \multicolumn{3}{|l|}{$95 \%$ CI } & \multicolumn{3}{|c|}{$1.167-1.230$} & & & \multicolumn{2}{|c|}{$1.055-1.149$} \\
\hline \multicolumn{3}{|l|}{ SD } & \multicolumn{3}{|c|}{0.075} & & & \multicolumn{2}{|c|}{0.098} \\
\hline \multicolumn{3}{|l|}{ Range } & \multicolumn{3}{|c|}{$1.090-1.3722$} & & & \multicolumn{2}{|c|}{$0.859-1.21$} \\
\hline
\end{tabular}

Mean alpha values in Maturational Stage I and II and alpha values of each subject in Maturational Stage I, respectively, Maturational Stage II. There was one subject with a double measurement in each age category; one subject had a double measurement in MS I, but only one measurement in MS II; one subject had one measurement in MS I and a double measurement in MS II; one subject had one measurement in each MS; four subjects had a single measurement in MS I; and thirteen subjects had double measurements in one category

maturational states MS I versus MS II was compared using $t$ test after testing for normality. Secondly, a linear regression analysis of the entire dataset of all measurements (accounting for age, sex, and maturational state) was performed to detect developmental differences in $\alpha$. Thirdly, a random-effects generalized least squares (GLS) regression analysis was performed for the statistical description of the combined cross-sectional and longitudinal dataset of $\alpha$, which accounted for age, sex, maturational state, and the presence of multiple measurements per subject (I and II). The repeatability of the measurements within 14 days was further illustrated by Bland and Altman plots (1986). The software used for the statistical analyses was STATA ${ }^{\circledR}$ v.10.0 for Windows.

\section{Results}

Patient characteristics of the study subjects are provided in Table 1. Table 2 provides a summary of all measured alpha values in both MSs. One subject (attending the study only at MS II) showed a pathological General Movement Assessment in the video, which is why it was excluded from all data analysis. The parameter $\alpha$ was $>0.5$ for all measurements $($ mean $=1.156, \mathrm{SD}=0.097)$, which indicated statistical persistence in each individual time series for acceleration. The cross-sectional comparison of the maturational stages I and II showed that alpha was significantly lower in MS II $(p=0.0003)$ (Table 2; Fig. 2a).

Regression analysis based on all measurements of $\alpha$ versus age is displayed in Fig. 2b. In Fig. 2b, corresponding individual longitudinal data series of the subjects are represented with lines. The linear regression analysis revealed a decreasing $\alpha$ with both increasing age over the entire measurement period (coefficient $=-0.00115,95 \%$ $\mathrm{CI}=-0.00182$ to $-0.00048, p=0.001)$ and ongoing maturation from MS I to MS II (coefficient $=-0.09619,95 \%$ $\mathrm{CI}=0.14881$ to $-0.04356, p=0.001)$ (Table 3 ).

GLS analysis was used due to hindered availability of subjects (relocation, acute illness etc....) leading to a heterogenic follow-up with a variable number of measurements (Table 2). Age and maturational stage showed no relevant interdependency (Table 4). Therefore, we assumed that the course of $\alpha$ in both MSs could be compared between both groups (Table 4). GLS analysis similarly showed a significant decrease of $\alpha$ with increasing age over the entire measurement period (Table 4).

In order to test short-term repeatability within 14 days, we displayed corresponding pairs in Fig. 3 in a BlandAltman illustration. None of the observed alpha values was outside confidence limits. 

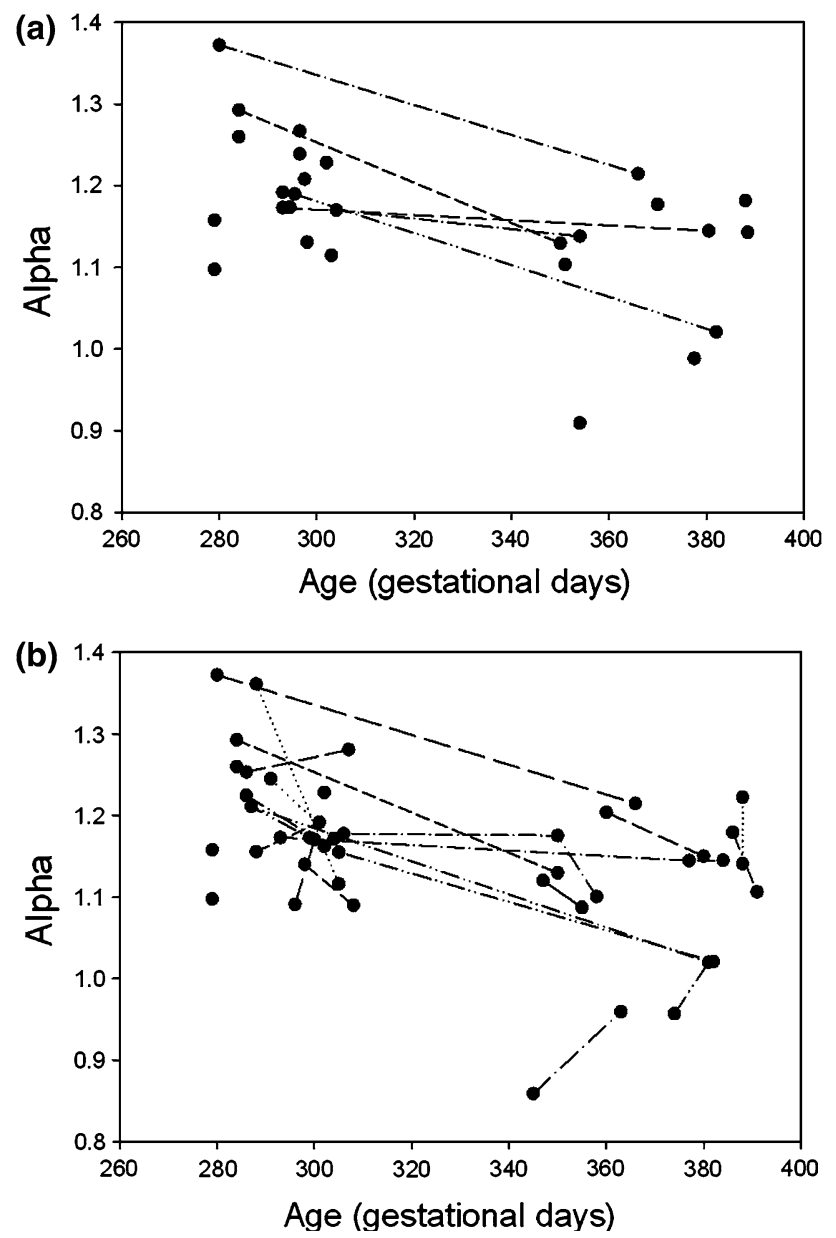

Fig. 2 a Cross-sectional dataset. The connecting lines illustrate the descriptive trend of decreasing $\alpha$ for subjects measured in both Maturational States I and II. For subjects with double measurements per maturational state mean $\alpha$ per maturational state is represented. b Longitudinal Dataset. Longitudinal dataset of all measured $\alpha$ plotted in relation to age at the time of the measurement. The connecting lines illustrate all of the longitudinal measurements per subject with a decreasing general trend of $\alpha$ over time

\section{Discussion}

\section{Summary}

The studies of Ohgi et al. and Gima et al. showed nonlinear, chaotic, and dynamic characteristics of spontaneous movements in premature infants (Ohgi et al. 2008) and qualitative changes in spontaneous movements of 8 healthy infants from 0 to 6 months (Gima et al. 2011). Using a similar method, our measurements also showed a significant fractal-type correlation in the acceleration of hand movements in infants and additionally revealed a significant alteration in motor behavior. Alpha decreased during a period of ongoing neurological maturation, in which the clinical assessment of spontaneous movements has been
Table 3 Linear regression analysis (all measurements)

\begin{tabular}{llll}
\hline & Coefficient & $95 \%$ CI & $\begin{array}{l}P \\
\text { value }\end{array}$ \\
\hline $\begin{array}{l}\text { Alpha versus age* } \\
\begin{array}{c}\text { Alpha versus } \\
\text { maturational stage }\end{array}\end{array}$ & -0.00115 & -0.00182 to -0.00048 & 0.001 \\
$\begin{array}{c}\text { Alpha versus age* } \\
\text { (maturational stage I) }\end{array}$ & -0.00230 & -0.00613 to 0.00014 & 0.060 \\
$\begin{array}{c}\text { Alpha versus age* } \\
\text { (maturational stage II) }\end{array}$ & 0.00146 & -0.00162 to 0.00455 & 0.331 \\
$\begin{array}{c}\text { Alpha versus sex } \\
\text { Alphe }\end{array}$ & 0.00678 & -0.05317 to 0.06673 & 0.821 \\
\hline
\end{tabular}

Alpha related to gestational age and maturational stage (linear regression analysis). There was a significant general decrease of $\alpha$ with increasing age as well as a significant decrease in $\alpha$ from Maturational Stage I to Maturational Stage II

* Age was defined as days after first day of last menstrual period (gestational age). CI Confidence interval

Table 4 GLS Model

\begin{tabular}{|c|c|c|c|}
\hline & Coefficient & $95 \%$ CI & $\begin{array}{l}P \\
\text { value }\end{array}$ \\
\hline $\begin{array}{l}\text { Alpha related to age* } \\
\text { clustered with matu- } \\
\text { rational stage }\end{array}$ & -0.00115 & -0.00181 to -0.00050 & 0.001 \\
\hline $\begin{array}{l}\text { Alpha related to age* } \\
\text { clustered with num- } \\
\text { ber of measurements } \\
\text { per subject }\end{array}$ & -0.00126 & -0.00187 to -0.00066 & $<0.001$ \\
\hline
\end{tabular}

Alpha related to gestational age and Maturational Stage. The agerelated analysis, clustered with Maturational Stage, revealed a decreasing trend in the value of $\alpha$

* Age was defined as days after first day of last menstrual period (gestational age)

shown to be significant for the prediction of later neurological outcomes (Cioni et al. 1997).

Defining neurological maturation by a numerical parameter

The clinical assessment of motor behavior in infants plays an important role in clinical practice. It has been shown that particularly, the complex behavior of SMs in young infants can predict later neurological outcomes (Burger and Louw 2009; Cioni et al. 1997; Einspieler et al. 2004; Einspieler and Prechtl 2005; Spittle et al. 2008). The most widely used method to describe motor behavior in infants is the "General Movement Assessment" according to Prechtl (Einspieler et al. 2004; Einspieler and Prechtl 2005). With detailed visual observation, this method describes maturational changes of spontaneous motor activity in infants. However, this method is time-consuming and requires highly specialized observers. Furthermore, the method shows a very variable intra- and inter-observer repeatability 


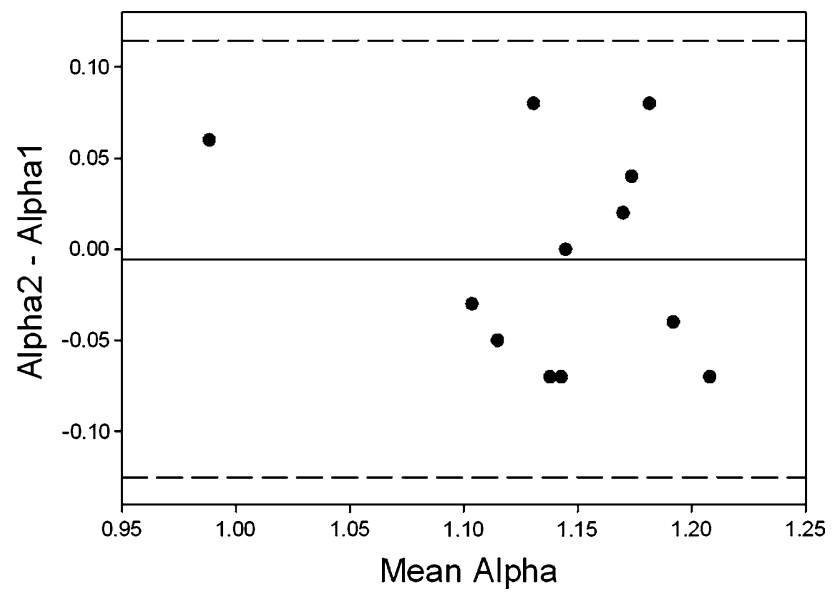

Fig. 3 Bland and Altman Plot. Six children in each maturational state were measured on two occasions within 14 days. The Bland-Altman representation descriptively shows a small amount of variability for repeated measurements within this interval. The continuous line represents the mean difference between both measurements, and the dotted lines represent two standard deviations of the difference between both measurements

(Bernhardt et al. 2011). Thus, there is a need for an automated and simplified method for detecting abnormal spontaneous motor activity in young infants.

Various quantitative methods have been proposed to evaluate the spontaneous motor activity in infants (Droit et al. 1996; Karch et al. 2008; Ohgi et al. 2007, 2008; Piek 2001; Vaal et al. 2000; van der Heide et al. 1999). Studies that examined the kinematic patterns of kicking movements found a difference between infants at risk for later cerebral palsy and healthy infants (Droit et al. 1996; Vaal et al. 2000; van der Heide et al. 1999). With three-dimensional kinematic analysis, it was also possible to detect maturational changes of spontaneous motor behavior in infants (Disselhorst-Klug et al. 2012). In addition, a recent study showed that the movement complexity of SMs in infants can be described by an automated approach (Karch et al. 2010). However, most of these studies used complex equipment, and therefore, the methods used are not necessarily suitable for a clinical setting.

Recently, Ohgi et al. (2008) performed accelerometric measurements of upper limb movements and demonstrated that spontaneous movements of premature infants have nonlinear, chaotic, dynamic characteristics, which are modified in infants with brain injury. Gima et al. (2011) also found nonlinear chaotic dynamics of spontaneous lower extremity movements and demonstrated time-dependent changes of spontaneous motor activity in 8 healthy infants. In our study, we used detrended fluctuation analysis to show evidence of fractal-type correlation in spontaneous motor activity in healthy infants (scaling exponent alpha). This method enabled us to characterize the complex waveforms of spontaneous movements in infants with one comprehensive parameter, which was observer-independent and reproducible over a short time period. We also demonstrated a change in $\alpha$ with maturation during early infancy, a period where significant changes of motor activity occur. The changing of $\alpha$ namely goes together with the transition of writhing movements to fidgety movements. In neither our study nor in the studies of Ohgi et al. and Gima et al., the predictive value for later neurological impairment was examined. Future studies shall correlate simple signals derived from a low cost accelerometer time series with long-term clinical neurological outcome.

As a complementary method to existing camera-based methods, automatically assessed acceleration signals with fractal-type correlation properties could help screening infants at risk for neurological impairment. Broader comparison of the known alpha coefficient values within other settings like gait studies (Hausdorff et al. 1999; Dingwell and Cusumano 2010) is necessary before placing the results in the context of this cohort.

Potential links to gait control mechanisms

The question arises as to whether our observations regarding the changes in the value of $\alpha$ could be linked to later motor development and gait control mechanisms. Previous studies of gait control mechanisms in adults (Hausdorff et al. 1995, 1996, 1997, 1999) showed an analog fractal-like behavior. Hausdorff et al. (1995) studied the stride interval in healthy adults and found evidence of long-range, self-similar correlations. In a later study, the same research group examined children and adults in different age groups and showed that the fractal-type long-range correlation of the stride interval in gait changes with age (Hausdorff et al. 1999). Our findings, showing a decreasing $\alpha$ with increasing age during early infancy, were consistent with and even complementary to their observations. Considering the results of Hausdorff et al. (1999), it would be interesting to investigate whether a fluent transition from fractal properties of spontaneous movements in infants to gait mechanisms in children and adults could be found, and whether this transition could be visualized with a continuous course of $\alpha$ as a function of age. To place the results of our study in this context and to explain these complex long-term age-related changes during neurological maturation, ongoing and future longitudinal follow-up studies should include computer simulations with broader datasets.

Limitations of the method

In an optimal setting, several longitudinal observations in the first month of life would have been performed. For different reasons (including relocation and illness), not all parents were willing to participate in the study at various time 
points. Thus, we had to analyze a combined cross-sectional and longitudinal dataset (Table 3). To ensure the robustness of the findings, we used different statistical methods (crosssectional age group comparison, linear regression analysis, random-effects generalized-least-squares regression analysis) to detect developmental differences in these subjects. All of the methods consistently showed a decrease of $\alpha$ with age, which supported the robustness and physiological relevance of the finding.

Furthermore, it must be noted that the pattern of hand movements may be influenced by the accelerometer on the infant's hand. The accelerometers weight is minimal, but the possible influence of the accelerometer by loss of movement intensity and by the child suckling on the tape around the accelerometer cannot be excluded. This limitation could have caused a bias due to varying hand-mouth contacts in MS I compared to MS II. To avoid disruptive suckling movements, future studies should place the accelerometers on the infant's feet or even use more than one accelerometer at the lower and the upper extremities simultaneously.

Primarily, the accelerometer was fixed to the infant's left hand. However, to ascertain a good fixation of the sensor, in 15 measurements, the accelerometer was placed on the right hand because of left-sided not removable baby wristbands. As only target-directed movements should show a right-hand dominance (Ottaviano et al. 1989), we did not insist on adapting the sensor always to the left hand. Future studies should investigate both sides in parallel to definitely rule out a hand preference. Particularly, in MS I, the moment of measurement was mainly defined by the private time schedule of mothers and infants at the maternity ward. Infants (neonates) needed to be breastfed or fell asleep during the $1 \mathrm{~h}$ measurement period; thus, lack of movement while sleeping and interaction with the mother during feeding may have caused a certain bias. To ascertain a $1 \mathrm{~h}$ lasting measurement with minimal length to apply DFA, an adjustment for different behavioral states (sleeping, drinking, sucking) was not possible.In four subjects, disconnection of the wire during ongoing measurement was shortening the record time to less than one hour. These measurements were excluded from the data analysis.

Various studies have described the characteristics of SMs in infants with respect to their timing. For example, the kicking frequency of infants has been determined to be between 4 and 80 times per minute (Piek and Carman 1994). Bos et al. described the trajectories of SMs in a quantitative manner and found that General Movement sequences occurred between 4 and 5 times per $10 \mathrm{~min}$ with a duration of 20-30 s. Other movement sequences such as isolated hand movements and twitches occurred more frequently (5-30 times per $10 \mathrm{~min}$ and 10-30 times per $10 \mathrm{~min}$, respectively) but were shorter in duration (Bos et al. 1997). The main information content of spontaneous movements is estimated below $3 \mathrm{~Hz}$ which is in principle in a safe range. Nevertheless, for small window sizes of the DFA, we may have a potential error due to the low sampling frequency. However, as a result of our simulation study (see Appendix 3), this error most likely won't affect the calculation of changes in the values of $\alpha$. Future faster technology with even smaller and more sensitive accelerometers should aim for higher sampling resolutions and accuracy.

\section{Conclusion and outlook}

We demonstrated that motor activity in infants shows fractal-type correlation, indicating that single movement accelerations are not independent of previous movement accelerations - in terms of a deterministic motor system sensitive to past behavior. Correlation properties were described by the parameter alpha, which is sensitive to motor developmental changes. As such the clinical impact of our findings is particularly relevant because these simple methods are potentially useful for detecting maturation of motor behavior during a time window in which clinical assessment of spontaneous movements has been shown to be predictive for later neurological outcome. The technique could be particularly useful for future clinical studies aimed at identifying infants with a high risk of impaired neurological outcome in an observer-independent manner with little effort. Furthermore, the methods could be used to investigate the effect of early intervention/therapy in larger cohorts of high-risk infants in a standardized setting.

Acknowledgments We would like to thank PD Dr. Matthias Nelle (Department of Neonatology, University Children's Hospital, Bern Switzerland), Brigitte Püntener and Prof. Dr. Lorenz Radlinger (University of Applied Sciences, Health, Bern, Switzerland) for their support.

Conflict of interest The authors declare that they have no conflict of interest.

\section{Appendix 1: Detrended fluctuation analysis (DFA)}

Detrended fluctuation analysis has been introduced in medical research to assess vital signals and biofeedback systems. DFA is an extension of fluctuation analysis of non-stationary time series (a signal whose mean and variance dynamics change over time) of stochastic processes to determine self-affinity of the signal and uncover fractal like properties. DFA was implemented according to Peng et al. (1995) as follows: The time series was first integrated and then divided into non-overlapping windows of size $n$. For each window, a least square first-order approximation was 
obtained which represents the "trend" in the signal. This first approximation of the integrated signal was denoted $y_{-} n(k)$. By subtracting $y_{-} n(k)$ from the integrated signal $y(k)$, the detrended signal was obtained. This is the approximation error $e_{n}(k)$ for which the root mean squared error was obtained and denoted

$F_{n}=\sqrt{\frac{1}{N} \sum_{k=1}^{N} e_{n}(k)^{2}}$

where $N$ is the number of intervals in the signal. Fn was obtained for several different window lengths $n$. With increasing window length, the approximation error increased: Log (n) versus $\log (\mathrm{Fn})$ gave a monotonically increasing relation that in the context of physiological signals has been found to be linear with slope alpha. Previous research has provided evidence for fractal characteristics in the signal: Fn and $n$ are related via a power-law, thus the linear characteristic of the $\log -\log$ relation. This scaling behaviour can then be precisely characterized by the value of alpha.

While Peng et al. (1993) have generally interpreted DFA as a measure of long-range correlations, recently Dingwell et al. (2010) and Maraun et al. (2004) proposed the interpretation of DFA as an indicator of persistence and anti-persistence (see "Introduction"). Herein we have followed their approach, thus, our results are interpretated in terms of persistence and anti-persistence of the acceleration time series.

An accelerometer time series with an $\alpha$ of 0.5 indicates a system that is not deterministic and is prone to instabilities, whereas higher $\alpha$ value implies more deterministic behavior with stronger correlations between an accelerated movement and its preceding accelerated movements (=persistence of an acceleration time series).

\section{Appendix 2: Statistical tests using surrogate time series}

Following Dingwell and Cusumano (2010), we generated for each measured time series one thousand randomly shuffled surrogates by independently permuting the order of the values in the time series (Hausdorff et al. 1995; Gates and Dingwell 2007; Theiler et al. 1992). These surrogates were used to test the hypothesis that the participants controlled their movements by sending signals to their muscles that

Table 5 Shuffling surrogate analysis MS I

\begin{tabular}{|c|c|c|c|c|c|c|c|c|c|c|}
\hline SID & Measurement & Alpha & $\begin{array}{l}p \text { value_Phase_ } \\
\text { Randomized }\end{array}$ & $\begin{array}{l}\text { Corrected_ } p \text { value_} \\
\text { Phase_Randomized }\end{array}$ & Min & $\begin{array}{l}\text { First } \\
\text { quartile }\end{array}$ & Median & Mean & $\begin{array}{l}\text { Third } \\
\text { quartile }\end{array}$ & Max \\
\hline 2 & 1 & 1.245 & $<0.001$ & $<0.028$ & 0.489 & 0.500 & 0.503 & 0.503 & 0.506 & 0.516 \\
\hline 2 & 2 & 1.171 & $<0.001$ & $<0.028$ & 0.489 & 0.500 & 0.503 & 0.503 & 0.507 & 0.513 \\
\hline 4 & 1 & 1.225 & $<0.001$ & $<0.028$ & 0.487 & 0.499 & 0.503 & 0.503 & 0.506 & 0.518 \\
\hline 4 & 2 & 1.155 & $<0.001$ & $<0.028$ & 0.494 & 0.500 & 0.503 & 0.503 & 0.506 & 0.515 \\
\hline 5 & 1 & 1.091 & $<0.001$ & $<0.028$ & 0.491 & 0.501 & 0.503 & 0.503 & 0.506 & 0.515 \\
\hline 5 & 2 & 1.170 & $<0.001$ & $<0.028$ & 0.492 & 0.500 & 0.503 & 0.503 & 0.506 & 0.516 \\
\hline 7 & 1 & 1.097 & $<0.001$ & $<0.028$ & 0.491 & 0.499 & 0.503 & 0.503 & 0.506 & 0.514 \\
\hline 8 & 1 & 1.228 & $<0.001$ & $<0.028$ & 0.491 & 0.499 & 0.502 & 0.502 & 0.506 & 0.514 \\
\hline 9 & 1 & 1.361 & $<0.001$ & $<0.028$ & 0.490 & 0.499 & 0.502 & 0.502 & 0.505 & 0.515 \\
\hline 9 & 2 & 1.116 & $<0.001$ & $<0.028$ & 0.493 & 0.500 & 0.503 & 0.503 & 0.506 & 0.517 \\
\hline 10 & 1 & 1.140 & $<0.001$ & $<0.028$ & 0.491 & 0.499 & 0.502 & 0.502 & 0.505 & 0.513 \\
\hline 10 & 2 & 1.090 & $<0.001$ & $<0.028$ & 0.490 & 0.499 & 0.502 & 0.502 & 0.505 & 0.517 \\
\hline 12 & 1 & 1.158 & $<0.001$ & $<0.028$ & 0.491 & 0.500 & 0.503 & 0.503 & 0.506 & 0.520 \\
\hline 13 & 1 & 1.162 & $<0.001$ & $<0.028$ & 0.490 & 0.499 & 0.502 & 0.503 & 0.507 & 0.518 \\
\hline 13 & 2 & 1.178 & $<0.001$ & $<0.028$ & 0.495 & 0.501 & 0.503 & 0.504 & 0.507 & 0.515 \\
\hline 15 & 1 & 1.260 & $<0.001$ & $<0.028$ & 0.493 & 0.500 & 0.503 & 0.503 & 0.506 & 0.513 \\
\hline 17 & 1 & 1.292 & $<0.001$ & $<0.028$ & 0.489 & 0.500 & 0.504 & 0.504 & 0.508 & 0.515 \\
\hline 3 & 1 & 1.156 & $<0.001$ & $<0.028$ & 0.492 & 0.498 & 0.502 & 0.502 & 0.505 & 0.517 \\
\hline 3 & 2 & 1.192 & $<0.001$ & $<0.028$ & 0.491 & 0.499 & 0.503 & 0.502 & 0.506 & 0.517 \\
\hline 19 & 1 & 1.211 & $<0.001$ & $<0.028$ & 0.493 & 0.500 & 0.502 & 0.502 & 0.505 & 0.517 \\
\hline 19 & 2 & 1.173 & $<0.001$ & $<0.028$ & 0.492 & 0.500 & 0.503 & 0.503 & 0.506 & 0.512 \\
\hline 20 & 1 & 1.372 & $<0.001$ & $<0.028$ & 0.493 & 0.500 & 0.503 & 0.503 & 0.505 & 0.514 \\
\hline 21 & 1 & 1.173 & $<0.001$ & $<0.028$ & 0.493 & 0.500 & 0.503 & 0.503 & 0.506 & 0.515 \\
\hline 22 & 1 & 1.253 & $<0.001$ & $<0.028$ & 0.487 & 0.499 & 0.503 & 0.503 & 0.506 & 0.518 \\
\hline 22 & 2 & 1.281 & $<0.001$ & $<0.028$ & 0.490 & 0.500 & 0.503 & 0.503 & 0.507 & 0.515 \\
\hline
\end{tabular}


Table 6 Shuffling surrogate analysis MS II

\begin{tabular}{|c|c|c|c|c|c|c|c|c|c|c|}
\hline SID & Measurement & Alpha & $\begin{array}{l}p \text { value_Phase_ } \\
\text { Randomized }\end{array}$ & $\begin{array}{l}\text { Corrected_ } p \text { value_} \\
\text { Phase_Randomized }\end{array}$ & Min & $\begin{array}{l}\text { First } \\
\text { quartile }\end{array}$ & Median & Mean & $\begin{array}{l}\text { Third } \\
\text { quartile }\end{array}$ & Max \\
\hline 1 & 3 & 1.120 & $<0.001$ & $<0.021$ & 0.491 & 0.500 & 0.504 & 0.503 & 0.507 & 0.513 \\
\hline 1 & 4 & 1.087 & $<0.001$ & $<0.021$ & 0.492 & 0.500 & 0.503 & 0.503 & 0.507 & 0.513 \\
\hline 4 & 3 & 1.021 & $<0.001$ & $<0.021$ & 0.493 & 0.500 & 0.504 & 0.503 & 0.507 & 0.515 \\
\hline 6 & 3 & 0.859 & $<0.001$ & $<0.021$ & 0.494 & 0.499 & 0.502 & 0.503 & 0.507 & 0.513 \\
\hline 6 & 4 & 0.959 & $<0.001$ & $<0.021$ & 0.490 & 0.499 & 0.502 & 0.502 & 0.505 & 0.519 \\
\hline 11 & 3 & 1.179 & $<0.001$ & $<0.021$ & 0.487 & 0.499 & 0.503 & 0.503 & 0.507 & 0.518 \\
\hline 11 & 4 & 1.106 & $<0.001$ & $<0.021$ & 0.494 & 0.501 & 0.504 & 0.504 & 0.507 & 0.515 \\
\hline 13 & 3 & 1.175 & $<0.001$ & $<0.021$ & 0.491 & 0.500 & 0.503 & 0.503 & 0.507 & 0.514 \\
\hline 13 & 4 & 1.101 & $<0.001$ & $<0.021$ & 0.492 & 0.500 & 0.503 & 0.503 & 0.507 & 0.514 \\
\hline 14 & 3 & 1.204 & $<0.001$ & $<0.021$ & 0.486 & 0.498 & 0.503 & 0.502 & 0.506 & 0.516 \\
\hline 14 & 4 & 1.150 & $<0.001$ & $<0.021$ & 0.491 & 0.500 & 0.503 & 0.503 & 0.506 & 0.515 \\
\hline 16 & 3 & 1.141 & $<0.001$ & $<0.021$ & 0.490 & 0.500 & 0.503 & 0.503 & 0.507 & 0.514 \\
\hline 16 & 4 & 1.222 & $<0.001$ & $<0.021$ & 0.492 & 0.499 & 0.503 & 0.503 & 0.507 & 0.517 \\
\hline 17 & 3 & 1.130 & $<0.001$ & $<0.021$ & 0.493 & 0.499 & 0.504 & 0.503 & 0.507 & 0.513 \\
\hline 18 & 3 & 0.957 & $<0.001$ & $<0.021$ & 0.490 & 0.500 & 0.502 & 0.502 & 0.505 & 0.511 \\
\hline 18 & 4 & 1.020 & $<0.001$ & $<0.021$ & 0.491 & 0.500 & 0.503 & 0.503 & 0.506 & 0.516 \\
\hline 20 & 3 & 1.215 & $<0.001$ & $<0.021$ & 0.491 & 0.499 & 0.503 & 0.503 & 0.506 & 0.516 \\
\hline 21 & 3 & 1.145 & $<0.001$ & $<0.021$ & 0.494 & 0.500 & 0.504 & 0.504 & 0.508 & 0.513 \\
\hline 21 & 4 & 1.145 & $<0.001$ & $<0.021$ & 0.491 & 0.500 & 0.503 & 0.503 & 0.507 & 0.516 \\
\hline 16 & 3 & 1.141 & $<0.001$ & $<0.021$ & 0.490 & 0.500 & 0.503 & 0.503 & 0.507 & 0.514 \\
\hline 16 & 4 & 1.222 & $<0.001$ & $<0.021$ & 0.492 & 0.499 & 0.503 & 0.503 & 0.507 & 0.517 \\
\hline
\end{tabular}

are temporally independent from previous signals. The distribution of alpha values displayed by the surrogate time series clearly allows for a rejection of this hypothesis for each participant at a significance level of $\alpha=0.05$ (see Tables 5, 6), after correcting the $p$ values for multiple comparison using Bonferroni's method.

Dingwell and Cusumano (2010) demonstrated that phaserandomized surrogates of stride-length time series and of stride-time time series obtained from young healthy adults walking on a motorized treadmill cannot be distinguished from the original time series in terms of detrended fluctuation analysis. Thereby, the hypothesis cannot be rejected, that subjects independently choose their stride-time and stride-length as temporally correlated auto-regressive (AR) process (Theiler et al. 1992; Schreiber and Schmitz 2000), or more generally as nearly equivalent auto-regressive moving-average (ARMA) process (Theiler et al., p. 81). We investigated to what extent this result applies to time series of motor acceleration measurements in healthy infants (at 40-44 and 50-55 weeks gestational age). Accordingly, we generated for each measured time series one thousand phase-randomized surrogates. That is, for each time series, we computed the Fourier transform, randomized the phase spectrum, and then computed the inverse Fourier transform (Theiler et al. 1992; Schreiber and Schmitz 2000; Dingwell and Cusumano 2000). These surrogates preserve the power spectra and auto-correlation properties of each original time series, thus preserving their statistical persistence.

Based on our results, for almost all participants, the aforementioned hypothesis, now concerning the subjects' limb acceleration patterns, cannot be rejected. Tables 7 and 8 list the resulting $p$ values from our statistical analysis using phase-randomized surrogate data for MS I and MS II.

The present work focuses on statistically significant changes in the alpha value of time series of motor acceleration measurements taken at 40-44 and at 50-55 weeks of gestational age and their potential interpretation as an indicator of maturational changes of motor behavior. However, we considered it pertinent to conduct similar tests as the ones performed in Dingwell and Cusumano (2010) in order to investigate potential analogies in the properties of the time series under consideration.

All calculations were conducted in R (R Core Team 2012).

\section{Appendix 3: Sampling frequency issues during measurements}

In order to study potential issues with the method of detrended fluctuation analysis that could arise from a low 
Table 7 Phase-randomized surrogate analysis MS I

\begin{tabular}{|c|c|c|c|c|c|c|c|c|c|c|}
\hline SID & Measurement & Alpha & $\begin{array}{l}p \text { value_Phase } \\
\text { Randomized }\end{array}$ & $\begin{array}{l}\text { Corrected_ } p \text { value } \\
\text { Phase_Randomized }\end{array}$ & Min & $\begin{array}{l}\text { First } \\
\text { quartile }\end{array}$ & Median & Mean & $\begin{array}{l}\text { Third } \\
\text { quartile }\end{array}$ & Max \\
\hline 2 & 1 & 1.245 & $<0.001$ & $<0.028$ & 1.257 & 1.266 & 1.269 & 1.269 & 1.271 & 1.279 \\
\hline 2 & 2 & 1.171 & 0.002 & 0.056 & 1.170 & 1.180 & 1.182 & 1.182 & 1.185 & 1.194 \\
\hline 4 & 1 & 1.225 & 0.358 & 1.000 & 1.213 & 1.221 & 1.223 & 1.223 & 1.226 & 1.233 \\
\hline 4 & 2 & 1.155 & 0.500 & 1.000 & 1.143 & 1.153 & 1.155 & 1.155 & 1.157 & 1.164 \\
\hline 5 & 1 & 1.091 & 0.160 & 1.000 & 1.075 & 1.085 & 1.088 & 1.088 & 1.090 & 1.098 \\
\hline 5 & 2 & 1.170 & 0.253 & 1.000 & 1.156 & 1.165 & 1.168 & 1.168 & 1.170 & 1.181 \\
\hline 7 & 1 & 1.097 & 0.104 & 1.000 & 1.081 & 1.091 & 1.093 & 1.093 & 1.095 & 1.105 \\
\hline 8 & 1 & 1.228 & 0.308 & 1.000 & 1.215 & 1.224 & 1.226 & 1.226 & 1.229 & 1.237 \\
\hline 9 & 1 & 1.361 & $<0.001$ & $<0.028$ & 1.363 & 1.373 & 1.375 & 1.375 & 1.379 & 1.388 \\
\hline 9 & 2 & 1.116 & 0.007 & 0.196 & 1.113 & 1.122 & 1.125 & 1.125 & 1.127 & 1.138 \\
\hline 10 & 1 & 1.140 & 0.400 & 1.000 & 1.131 & 1.139 & 1.141 & 1.141 & 1.143 & 1.155 \\
\hline 10 & 2 & 1.090 & 0.224 & 1.000 & 1.083 & 1.090 & 1.092 & 1.092 & 1.094 & 1.102 \\
\hline 12 & 1 & 1.158 & 0.161 & 1.000 & 1.149 & 1.159 & 1.161 & 1.161 & 1.163 & 1.172 \\
\hline 13 & 1 & 1.162 & 0.337 & 1.000 & 1.154 & 1.161 & 1.164 & 1.164 & 1.166 & 1.174 \\
\hline 13 & 2 & 1.178 & 0.048 & 1.000 & 1.173 & 1.181 & 1.184 & 1.184 & 1.186 & 1.193 \\
\hline 15 & 1 & 1.260 & $<0.001$ & $<0.028$ & 1.233 & 1.241 & 1.244 & 1.244 & 1.246 & 1.256 \\
\hline 17 & 1 & 1.292 & 0.072 & 1.000 & 1.275 & 1.285 & 1.287 & 1.287 & 1.290 & 1.298 \\
\hline 3 & 1 & 1.156 & 0.439 & 1.000 & 1.143 & 1.154 & 1.156 & 1.156 & 1.159 & 1.168 \\
\hline 3 & 2 & 1.192 & 0.003 & 0.084 & 1.191 & 1.199 & 1.202 & 1.202 & 1.204 & 1.213 \\
\hline 19 & 1 & 1.211 & 0.002 & 0.056 & 1.209 & 1.219 & 1.221 & 1.221 & 1.224 & 1.232 \\
\hline 19 & 2 & 1.173 & 0.372 & 1.000 & 1.160 & 1.169 & 1.172 & 1.172 & 1.174 & 1.182 \\
\hline 20 & 1 & 1.372 & 0.179 & 1.000 & 1.363 & 1.373 & 1.376 & 1.376 & 1.379 & 1.388 \\
\hline 21 & 2 & 1.173 & 0.267 & 1.000 & 1.161 & 1.168 & 1.171 & 1.171 & 1.173 & 1.182 \\
\hline 22 & 1 & 1.253 & 0.322 & 1.000 & 1.237 & 1.249 & 1.252 & 1.252 & 1.254 & 1.266 \\
\hline 22 & 2 & 1.281 & 0.483 & 1.000 & 1.270 & 1.278 & 1.280 & 1.281 & 1.283 & 1.292 \\
\hline
\end{tabular}

sampling frequency during the measurement, we conducted a simulation study. We investigated the effect of applying detrended fluctuation analysis to a time series obtained from an "original" time series by sampling the time series with a lower sampling rate. This sampling procedure boils down to leaving out one or more consecutive values from the original time series in a periodic manner.

The "original" time series consisted of pink noise generated via simulation. It can be theoretically shown that the alpha value obtained from such a time series should be approximately equal to 1 . We generated one thousand such time series and with each one we conducted the sampling procedure with a fixed sampling rate. Different sampling rates were used in order to assess the strength of the effect as the sampling frequency is lowered. For a given sampling rate, the aforementioned procedure yielded one thousand shorter time series. We then compared the alpha value of the original time series with the alpha value of the series obtained via the sampling procedure. We observed a change of the alpha value, which was consistently in the same direction. The distribution of the differences in the alpha values is displayed in Fig. 4.

Since the sampling procedure generates shorter time series, we also generated additional one thousand pink noise time series of the same length as the ones resulting from the sampling procedure. We compared the distributions of the alpha values in each of the two groups observing a statistically significant difference ( $p$ values $\leq 1.705 \mathrm{e}-10$, Mann-Whitney test, $p$ values $\leq 1.866 \mathrm{e}-8$, KolmogorovSmirnov test).

The mean strength of the effect varied with the sampling frequency used. The introduced relative error (in percentage) ranges from $4.65 \%$, when leaving out every other value, to $19.37 \%$, when only every hundredth value is considered (see Fig. 5).

Fortunately, the effect is consistent in direction, suggesting that the errors in the alpha values caused by a low sampling rate during measurement should not jeopardize the validity of our main results. Of central importance for our study is the change in the alpha values during development. While the interpretation of the actual absolute alpha 
Table 8 Phase-randomized surrogate analysis MS II

\begin{tabular}{|c|c|c|c|c|c|c|c|c|c|c|}
\hline SID & Measurement & Alpha & $\begin{array}{l}p \text { value_Phase_ } \\
\text { Randomized }\end{array}$ & $\begin{array}{l}\text { Corrected_p value_ } \\
\text { Phase_Randomized }\end{array}$ & Min & $\begin{array}{l}\text { First } \\
\text { quartile }\end{array}$ & Median & Mean & $\begin{array}{l}\text { Third } \\
\text { quartile }\end{array}$ & Max \\
\hline 1 & 3 & 1.120 & 0.286 & 1.000 & 1.106 & 1.116 & 1.118 & 1.118 & 1.120 & 1.129 \\
\hline 1 & 4 & 1.087 & 0.005 & 0.105 & 1.071 & 1.078 & 1.080 & 1.080 & 1.082 & 1.089 \\
\hline 4 & 3 & 1.021 & 0.014 & 0.294 & 0.997 & 1.009 & 1.012 & 1.012 & 1.014 & 1.023 \\
\hline 6 & 3 & 0.859 & 0.208 & 1.000 & 0.847 & 0.855 & 0.857 & 0.857 & 0.859 & 0.866 \\
\hline 6 & 4 & 0.959 & 0.078 & 1.000 & 0.953 & 0.962 & 0.964 & 0.964 & 0.966 & 0.976 \\
\hline 11 & 3 & 1.106 & 0.472 & 1.000 & 1.094 & 1.104 & 1.106 & 1.106 & 1.109 & 1.117 \\
\hline 11 & 4 & 1.179 & 0.443 & 1.000 & 1.167 & 1.176 & 1.179 & 1.179 & 1.181 & 1.191 \\
\hline 13 & 3 & 1.175 & 0.319 & 1.000 & 1.166 & 1.175 & 1.177 & 1.177 & 1.180 & 1.192 \\
\hline 13 & 4 & 1.101 & 0.504 & 1.000 & 1.086 & 1.098 & 1.101 & 1.100 & 1.103 & 1.112 \\
\hline 14 & 3 & 1.204 & 0.008 & 0.168 & 1.203 & 1.211 & 1.214 & 1.214 & 1.217 & 1.225 \\
\hline 14 & 4 & 1.150 & 0.093 & 1.000 & 1.144 & 1.152 & 1.155 & 1.155 & 1.157 & 1.165 \\
\hline 17 & 3 & 1.130 & 0.062 & 1.000 & 1.112 & 1.123 & 1.125 & 1.125 & 1.127 & 1.134 \\
\hline 18 & 3 & 0.957 & 0.499 & 1.000 & 0.946 & 0.954 & 0.957 & 0.957 & 0.959 & 0.970 \\
\hline 18 & 4 & 1.020 & 0.052 & 1.000 & 1.002 & 1.011 & 1.014 & 1.013 & 1.016 & 1.029 \\
\hline 20 & 3 & 1.215 & $<0.001$ & $<0.021$ & 1.143 & 1.153 & 1.155 & 1.155 & 1.158 & 1.167 \\
\hline 21 & 3 & 1.145 & 0.003 & 0.063 & 1.143 & 1.152 & 1.155 & 1.155 & 1.157 & 1.169 \\
\hline 21 & 4 & 1.145 & 0.436 & 1.000 & 1.132 & 1.143 & 1.145 & 1.145 & 1.148 & 1.156 \\
\hline 16 & 3 & 1.141 & 0.038 & 0.798 & 1.136 & 1.144 & 1.147 & 1.147 & 1.149 & 1.156 \\
\hline 16 & 4 & 1.222 & 0.206 & 1.000 & 1.214 & 1.223 & 1.225 & 1.225 & 1.227 & 1.237 \\
\hline
\end{tabular}

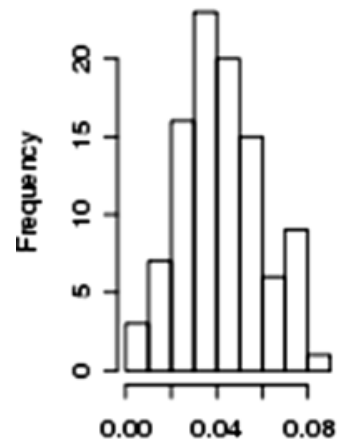

Absolute Enors

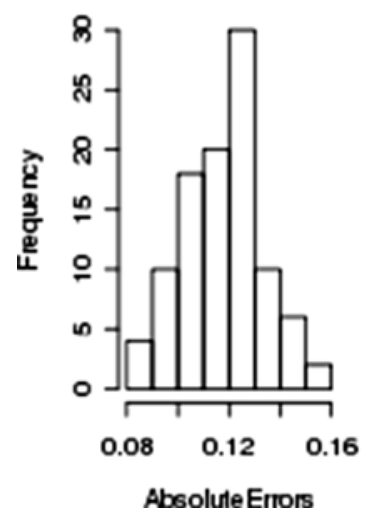

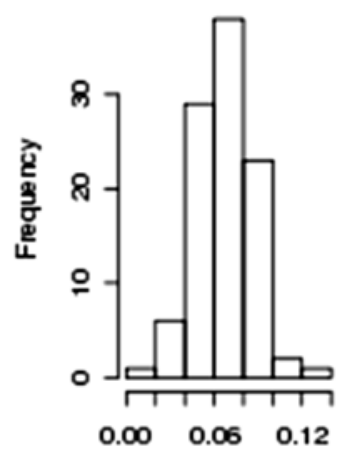

Absolute Errors

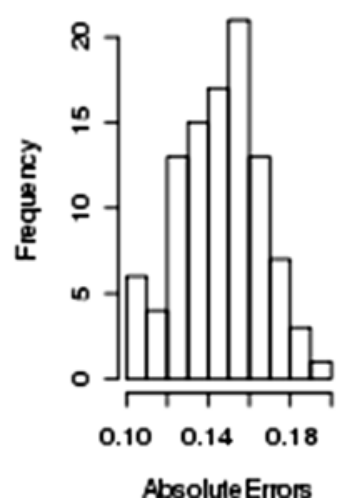

Absolute Errors
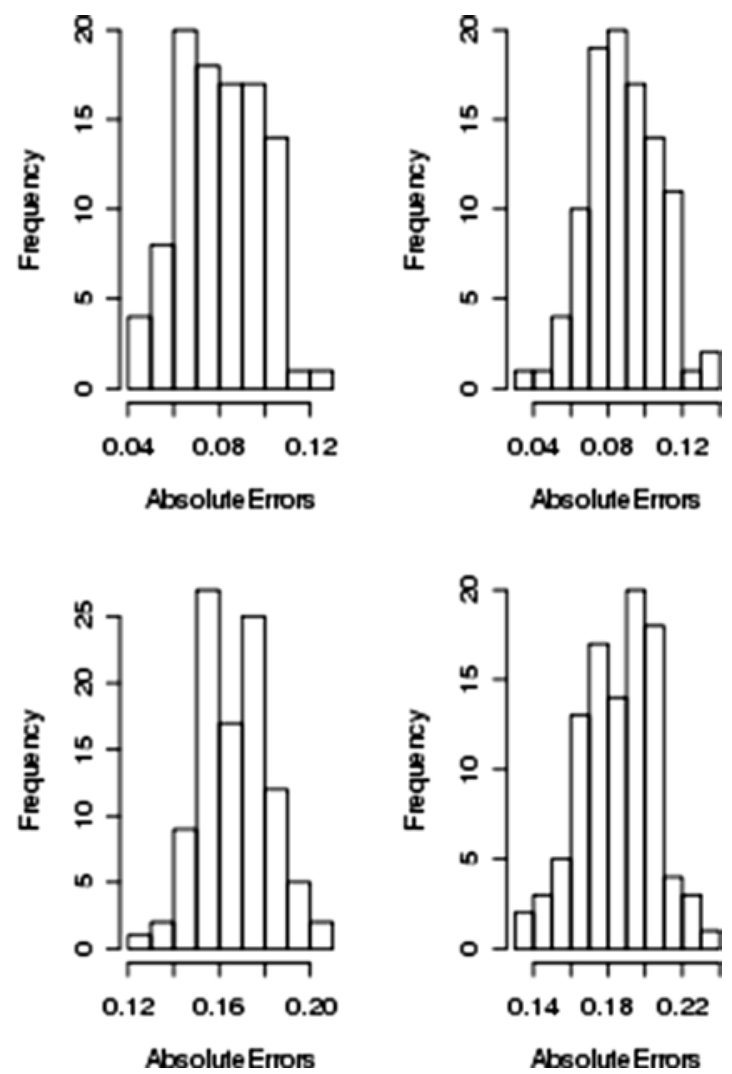

Fig. 4 Histograms of absolute errors due to subsampling. The distribution of the differences in the alpha values due to sampling frequency issues. Every panel corresponds to a different sampling fre- quency. The number of data points left out between recorded values are: Upper row, from left to right 1, 2, 3, 4. Lower Row, from left to right $10,20,50,100$ 


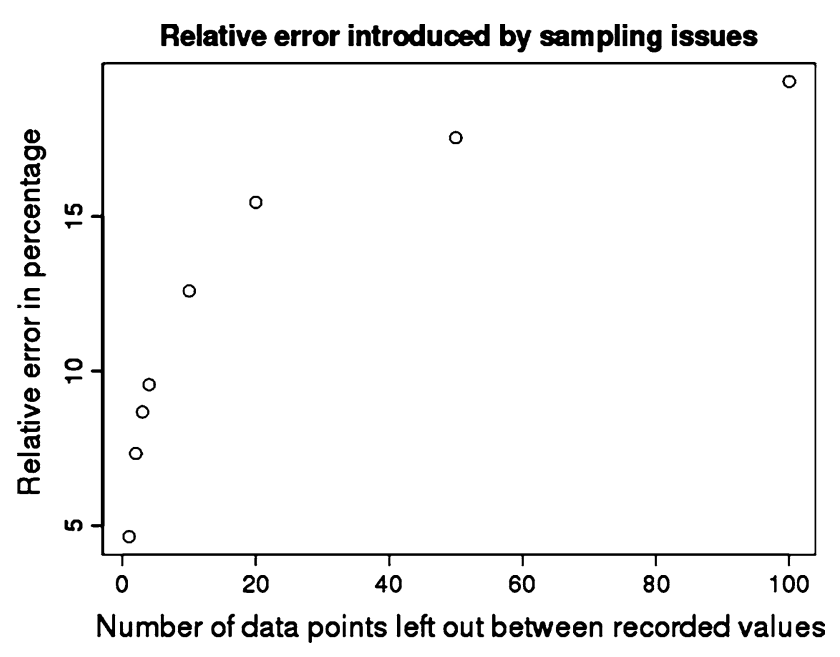

Fig. 5 Relative error due to subsampling

values might be interesting (and controversial, as pointed out in the literature, see, for instance, Maraun et al. 2004), we focused herein more on the change of this value during development. We believe that, as long as we consistently use the same sampling frequency during all measurements, the reported changes in the value of alpha properly quantify the changes in the statistical persistence and anti-persistence properties of the sampled time series. Consequently, the reported changes in the value of alpha might represent a legitimate way of systematically quantifying neurological maturation.

All calculations were conducted in R (R Core Team 2012).

\section{References}

Baldwin DN, Suki B, Pillow JJ, Roiha HL, Minocchieri S, Frey U (2004) Effect of sighs on breathing memory and dynamics in healthy infants. J Appl Physiol 97:1830-1839

Bernhardt I, Marbacher M, Hilfiker R, Radlinger L (2011) Inter- and intra-observer agreement of Prechtl's method on the qualitative assessment of general movements in preterm, term and young infants. Early Hum Dev 87(9):633-639

Bland JM, Altman DG (1986) Statistical methods for assessing agreement between two methods of clinical measurement. Lancet $1: 307-310$

Bos AF, van Loon AJ, Martijn A, van Asperen RM, Okken A, Prechtl HF (1997) Spontaneous motility in preterm, small-for-gestational age infants I. Quantitative aspects. Early Hum Dev 50:115-129

Burger M, Louw QA (2009) The predictive validity of general movements-a systematic review. Eur J Paediatr Neurol 13:408-420

Cernelc M, Suki B, Reinmann B, Hall GL, Frey U (2002) Correlation properties of tidal volume and end-tidal $\mathrm{O} 2$ and $\mathrm{CO} 2$ concentrations in healthy infants. J Appl Physiol 92:1817-1827

Cioni G, Prechtl HF, Ferrari F, Paolicelli PB, Einspieler C, Roversi MF (1997) Which better predicts later outcome in full-term infants: quality of general movements or neurological examination? Early Hum Dev 50:71-85
Constantine W, Percival D (2012) Fractal: Fractal Time Series Modeling and Analysis. $\mathrm{R}$ package version 1.1-1. http:// CRAN.R-project.org/package $=$ fractal

Dingwell JB, Cusumano JP (2000) Nonlinear time series analysis of normal and pathological human walking. Chaos 10:848-863

Dingwell JB, Cusumano JP (2010) Re-interpreting detrended fluctuation analyses of stride-to-stride variability in human walking. Gait Posture 32:348-353

Disselhorst-Klug C, Heinze F, Breitbach-Faller N, Schmitz-Rode T, Rau G (2012) Introduction of a method for quantitative evaluation of spontaneous motor activity development with age in infants. Exp Brain Res 218:305-313

Droit S, Boldrini A, Cioni G (1996) Rhythmical leg movements in low-risk and brain-damaged preterm infants. Early Hum Dev 44:201-213

Einspieler C, Prechtl HF (2005) Prechtl's assessment of general movements: a diagnostic tool for the functional assessment of the young nervous system. Ment Retard Dev Disabil Res Rev 11:61-67

Einspieler C, Prechtl HF, Ferrari F, Cioni G (2004) Prechtl's method on the qualitative assessment of general movements in preterm. Cambridge University Press, Term and Young Infants

Ferrari F, Cioni G, Prechtl HF (1990) Qualitative changes of general movements in preterm infants with brain lesions. Early Hum Dev 23:193-231

Gates DH, Dingwell JB (2007) Peripheral neuropathy does not alter the fractal dynamics of gait stride intervals. J Appl Physiol 102(3):965-971

Gima H, Ohgi S, Morita S, Karasuno H, Fujiwara T, Abe K (2011) A dynamical system analysis of the development of spontaneous lower extremity movements in newborn and young infants. $\mathrm{J}$ Physiol Anthropol 30(5):179-186

Goldberger AL, Amaral LA, Hausdorff JM, Ivanov PC, Peng CK, Stanley HE (2002) Fractal dynamics in physiology: alterations with disease and aging. Proc Natl Acad Sci USA 99(Suppl 1):2466-2472

Hausdorff JM, Peng CK, Ladin Z, Wei JY, Goldberger AL (1995) Is walking a random walk? Evidence for long-range correlations in stride interval of human gait. J Appl Physiol 78:349-358

Hausdorff JM, Purdon PL, Peng CK, Ladin Z, Wei JY, Goldberger AL (1996) Fractal dynamics of human gait: stability of longrange correlations in stride interval fluctuations. J Appl Physiol 80:1448-1457

Hausdorff JM, Mitchell SL, Firtion R, Peng CK, Cudkowicz ME, Wei JY, Goldberger AL (1997) Altered fractal dynamics of gait: reduced stride-interval correlations with aging and Huntington's disease. J Appl Physiol 82:262-269

Hausdorff JM, Zemany L, Peng C, Goldberger AL (1999) Maturation of gait dynamics: stride-to-stride variability and its temporal organization in children. J Appl Physiol 86:1040-1047

Karch D, Kim KS, Wochner K, Pietz J, Dickhaus H, Philippi H (2008) Quantification of the segmental kinematics of spontaneous infant movements. J Biomech 41:2860-2867

Karch D, Wochner K, Kim K, Philippi H, Hadders-Algra M, Pietz J, Dickhaus H (2010) Quantitative score for the evaluation of kinematic recordings in neuropediatric diagnostics. Detection of complex patterns in spontaneous limb movements. Methods Inf Med 49:526-530

Ligges U (2011) Tune R: Analysis of music. http://r-forge.r-project.org/ projects/tuner/

Maraun D, Rust HW, Timmer J (2004) Tempting long-memory—on the interpretation of DFA results. Nonlinear Process Geophys 11:495-503

Meinecke L, Breitbach-Faller N, Bartz C, Damen R, Rau G, Disselhorst-Klug C (2006) Movement analysis in the early detection 
of newborns at risk for developing spasticity due to infantile cerebral palsy. Hum Mov Sci 25:125-144

Ohgi S, Morita S, Loo KK, Mizuike C (2007) A dynamical systems analysis of spontaneous movements in newborn infants. J Mot Behav 39:203-214

Ohgi S, Morita S, Loo KK, Mizuike C (2008) Time series analysis of spontaneous upper-extremity movements of premature infants with brain injuries. Phys Ther 88:1022-1033

Ottaviano S, Guidetti V, Allemand F, Spinetoli B, Seri S (1989) Laterality of arm movement in full-term newborn. Early Hum Dev 19:3-7

Peng CK, Mietus J, Hausdorff JM, Havlin S, Stanley HE, Goldberger AL (1993) Long-range anticorrelations and non-gaussian behavior of the heartbeat. Phys Rev Lett 70:1343-1346

Peng CK, Havlin S, Stanley HE, Goldberger AL (1995) Quantification of scaling exponents and crossover phenomena in nonstationary heartbeat time-series. Chaos 5:82-87

Piek JP (2001) Is a quantitative approach useful in the comparison of spontaneous movements in fullterm and preterm infants? Hum Mov Sci 20:717-736

Piek JP, Carman R (1994) Developmental profiles of spontaneous movements in infants. Early Hum Dev 39:109-126

Prechtl HF (1974) The behavioural states of the newborn infant (a review). Brain Res 76:185-212

R Core Team (2012) R: A language and environment for statistical computing. R Foundation for Statistical Computing, Vienna, Austria (2012). ISBN 3-900051-07-0, http://www.R-project.org
Schreiber T, Schmitz A (2000) Surrogate time series. Physics 142(3-4):346-382

Spittle AJ, Doyle LW, Boyd RN (2008) A systematic review of the clinimetric properties of neuromotor assessments for preterm infants during the first year of life. Dev Med Child Neurol 50:254-266

Stern G, Beel J, Suki B, Silverman M, Westaway J, Cernelc M, Baldwin D, Frey U (2009) Long-range correlations in rectal temperature fluctuations of healthy infants during maturation. PLoS One 4:e6431

Thamrin C, Frey U (2009) Complexity and respiratory growth: a developing story. J Appl Physiol 106:753-754

Thamrin C, Stern G, Frey U (2010) Fractals for physicians. Paediatr Respir Rev 11:123-131

Theiler J, Eubank S, Longtin A, Galdrikian B, Farmer JD (1992) Testing for nonlinearity in time series: the method of surrogate data. Phys D 58(1-4):77-94

Vaal J, Van Soest AJ, Hopkins B, Sie LT, Van der Knaap MS (2000) Development of spontaneous leg movements in infants with and without periventricular leukomalacia. Exp Brain Res 135:94-105

Van der Heide J, Paolicelli PB, Boldrini A, Cioni G (1999) Kinematic and qualitative analysis of lower extremitymovements in preterm infants with brain lesions. Phys Ther 79:546-557 